\author{
Tracking intraoperative fluoroscopy utilization reduces radiation exposure during ureteroscopy \\ Ngo TC, Macleod LC, Rosenstein DI, Reese JH, Shinghal R \\ Department of Urology, Stanford University School of Medicine, Stanford, California 94305, USA \\ J Endourol. 2011; 25: 763-7
}

Purpose: Recent studies have demonstrated deleterious effects of ionizing radiation from diagnostic and therapeutic imaging procedures. One of the barriers to minimizing patient exposure is physician awareness. We prospectively studied whether providing surgeons with feedback on their fluoroscopy utilization would affect intraoperative fluoroscopy times.

Materials and Methods: In 2007, we prospectively began to track fluoroscopy usage for all urology cases. Nine months later, surgeons started to receive periodic reports with their mean fluoroscopy time compared with their peers. We reviewed all ureteroscopic cases for nephrolithiasis from the date tracking began (2006$2010, \mathrm{n}=311$ ). Using the initial 9-month period as a control, we studied the effect of providing feedback on mean fluoroscopy times in subsequent periods and analyzed patient factors that may affect radiation exposure. Results: Mean fluoroscopy times for unilateral ureteroscopy decreased by $24 \%$ after surgeons received feedback (2.74-2.08 minutes, $\mathrm{p}=0.002)$. On multivariate analysis, factors that independently predicted decreased fluoroscopy times included female sex $(p=0.02)$, stones in the distal ureter $(p=0.04)$, and if the surgeon had received feedback $(p=0.0004)$. Factors that increased fluoroscopy times included the presence of hydronephrosis $(p=0.001)$, use of a ureteral access sheath $(p=0.04)$, ureteral balloon dilation $(p=0.0001)$, and placement of a postoperative stent $(\mathrm{p}=0.002)$.

Conclusions: Providing surgeons with feedback on their fluoroscopy usage reduces patient and surgeon radiation exposure. Implementing such a tracking system requires minimal changes to existing operating room staff workflow. Further study is warranted to study the impact of this program on other procedures that utilize fluoroscopy in urology and other specialties.

\title{
Editorial Comment
}

This is an important study that could have major impact on radiation exposure - it is a worthy initiative that should be extended to shockwave lithotripsy, percutaneous nephrolithotomy, videourodynamics and beyond urology. It is important to note that fluoroscopy on-time is not the sole variable that the urologist can control to minimize radiation exposure. It would be useful to provide a more comprehensive educational tool, that included the appropriate positioning of the c-arm and image intensifier (distance from the patient skin, under-table configuration, avoiding angled trajectories), the appropriate use of last image hold and collimation, and other radiation-mitigating variables that can make the OR a safer place for patient and physician.

The inclusion of patients treated with an occlusion device in the second arm of the study is an important confounder to the study. It is feasible that an occlusion device could decrease operative time and fluoroscopy time if it prevented stone migration and the need for adjunctive flexible ureteroscopy. It may have been worthwhile to exclude these patients, and evaluate two cohorts treated with or without an occlusion device in the second phase of the study to evaluate the impact of the device.

Dr. Manoj Monga

Director, Stevan B. Streem Center for Endourology \& Stone Disease Glickman Urological \& Kidney Institute

The Cleveland Clinic

Cleveland, Ohio, USA

E-mail:endourol@yahoo.com 